\title{
Utilização do planejamento experimental em rede simplex no estudo de resíduo de rocha ornamental como filler para obtenção de máxima compacidade
}

\section{(Use of simplex lattice experimental design in the study of ornamental rock waste as filler to obtain maximum compaction)}

\author{
A.Z. Destefani, J. N. F. Holanda \\ Grupo de Materiais Cerâmicos, LAMAV-CCT, Universidade Estadual do Norte Fluminense Darcy Ribeiro, \\ Av. Alberto Lamego, 2000, Campos dos Goytacazes, RJ 28013-600 \\ andestefani@hotmail.com,holanda@uenf.br
}

\begin{abstract}
Resumo
A utilização de agregados industrializados vem crescendo ao longo dos anos para atender a grande demanda da construção civil devido ao crescimento econômico do país. O objetivo deste trabalho foi utilizar o planejamento experimental em Rede Simplex para avaliar o efeito da adição do resíduo de rocha ornamental como filler na composição de misturas ternárias (brita 0 , pó de pedra e resíduo), que levem a máxima compacidade (densidade seca aparente máxima). Foram tomados dezesseis pontos experimentais, cujos teores dos materiais utilizados variaram de 0 a 100\%. O modelo em rede simplex cúbico completo apresentou melhor ajuste aos resultados experimentais, o qual resulta em respostas estatisticamente mais adequadas para as composições estudadas. A superfície de resposta gerada indicou que a densidade seca aparente máxima de $2,0 \mathrm{~g} / \mathrm{cm}^{3}$ foi obtida para a composição ternária: $63 \%$ de brita $0 / 17 \%$ de pó de pedra/20\% de resíduo de rocha ornamental. Portanto, o uso de resíduo de rocha ornamental como filler em agregados para a construção civil pode ser uma alternativa viável para deposição final deste abundante resíduo de forma ambientalmente correta.
\end{abstract}

Palavras-chave: resíduo de rocha, filler, planejamento experimental, simplex, agregado.

\begin{abstract}
The use of industrial aggregates has grown over the years to meet the great demand of the civil construction due to the country's economical growth. The aim of this work was to use the experimental design in Simplex Lattice to evaluate the effect of the addition of ornamental rock waste as filler in the composition of ternary mixtures (crushed rock 0, stone powder, rock waste), leading to maximum compaction (maximum apparent dry density). Sixteen experimental points were taken, whose contents of the used materials ranged from 0 to $100 \%$. The complete cubic simplex model showed to best fit to the experimental results, which results in more statistically appropriated responses to the studied compositions. The response surface generated indicated that the maximum apparent dry density $\left(2.0 \mathrm{~g} / \mathrm{cm}^{3}\right)$ was obtained for the ternary composition: $63 \%$ crushed rock $0 / 17 \%$ stone powder/20\% ornamental rock waste. Therefore, the use of ornamental rock waste as filler in aggregates for civil construction can be a viable alternative for final deposition in an environmental correct way.
\end{abstract}

Keywords: rock waste, filler, experimental design, simplex, aggregate.

\section{INTRODUÇÃO}

Diversas propriedades dos materiais estão fortemente associadas ao empacotamento das partículas que o constituem. Em particular, empacotamentos densos são de alto interesse no processamento de materiais aplicados aos mais diversos setores tecnológicos como concretos, materiais cerâmicos, metalurgia do pó, entre outros [1,2]. O estudo do empacotamento de partículas está intimamente relacionado à definição do problema da correta seleção da proporção e do tamanho adequado dos materiais particulados, de tal forma que os vazios maiores sejam preenchidos com partículas menores, cujos vazios serão novamente preenchidos com partículas ainda menores e assim sucessivamente [3]. No caso dos produtos cimentícios a otimização do empacotamento das partículas pode ser feito por meio de adições minerais. Via de regra, as adições minerais são materiais silicosos finamente divididos, e normalmente adicionados ao concreto em quantidades relativamente grandes, que variam de 20 a $70 \%$ em massa do material cimentício total [4]. Em geral as mudanças nas propriedades de produtos cimentício confeccionados com aditivos minerais podem ser atribuídas aos efeitos físicos e químicos. Os efeitos físicos podem ser divididos em três: diluição do cimento Portland, efeito filler e nucleação. $\mathrm{O}$ fíller é uma adição mineral finamente dividida sem atividade química, ou seja, sua ação se resume a um efeito físico de empacotamento granulométrico. Neste contexto, pó de sílica, pó de pedra e calcário são materiais considerados 
como sendo fíller [5].

O Brasil detém uma expressiva indústria de rochas ornamentais espalhada nas diversas regiões do país. A produção brasileira de rochas ornamentais em 2007 foi de cerca de 8 milhões de toneladas [6]. A indústria de rochas ornamentais gera muita riqueza e desenvolvimento social no país com a criação de milhares de empregos diretos e indiretos. Por outro lado, a indústria de rochas ornamentais também gera enormes quantidades de resíduos sólidos poluentes [7-9]. Estes resíduos sólidos na maioria das vezes, principalmente no Brasil, são dispostos inadequadamente no meio ambiente, o qual resulta em poluição ambiental de difícil solução. De fato os resíduos de rochas ornamentais podem ser classificados de acordo com as normas ambientais brasileiras como sendo materiais de resíduos do tipo classe IIA - Não Inerte [10]. Esta situação é decorrente da possibilidade de solubilização de metais pesados como chumbo e cromo, além das presenças de ferro e manganês acima dos limites máximos permitidos para o ensaio de solubilização. Isto reforça a necessidade na busca de novas alternativas para a disposição final destes abundantes resíduos. A problemática dos resíduos sólidos é um dos grandes gargalos a ser enfrentado pela indústria de rochas ornamentais no século XXI. Trabalhos têm mostrado a possibilidade de reaproveitamento destes resíduos sólidos na fabricação de cerâmica vermelha e revestimentos cerâmicos para pisos [7, 8, 11-13]. No entanto, estes resíduos têm sido pouco estudados como material filler em materiais cimentícios. Isto é interessante devido os resíduos sólidos provenientes do beneficiamento de rochas ornamentais, em grande parte, apresentarem características químicas, mineralógicas, morfológicas e granulométricas com grande potencial para utilização deles como material fíller [14].

Este trabalho tem como objetivo utilizar o planejamento experimental em rede simplex, aplicado ao estudo de misturas, para avaliar o comportamento de empacotamento de misturas de três materiais (brita 0 , pó de pedra e resíduo de rocha ornamental) visando a sua utilização como agregado industrial.

\section{MATERIAIS E MÉTODOS}

Os concretos convencionais têm como constituintes o cimento Portland, agregados e água. A proposta deste trabalho é a elaboração do traço com a maior compacidade (densidade máxima aparente) utilizando os agregados industrializados brita 0 e pó de pedra, e o resíduo do beneficiamento de rochas ornamentais como fíller. Brita 0 e pó de pedra usados como agregados são provenientes da região de Campos dos Goytacazes, RJ. Estes materiais foram inicialmente submetidos a um processo de lavagem em peneira ABNT 200 e posteriormente por secagem em estufa a $105{ }^{\circ} \mathrm{C}$ por $24 \mathrm{~h}$. O resíduo de beneficiamento de rochas ornamentais é proveniente do município de Cachoeiro de Itapemirim, ES. A amostra de resíduo foi coletada obedecendo aos procedimentos de amostragem estabelecidos na norma ABNT NBR 10007 [15]. O resíduo foi coletado em estado seco ao ar em depósito anexo à empresa fornecedora. Após coleta, o resíduo foi submetido a secagem em estufa a $105^{\circ} \mathrm{C}$ por $24 \mathrm{~h}$, desagregado e homogeneizado para ser utilizado. Os materiais utilizados como agregados (brita 0 , pó de pedra e resíduo de rocha ornamental) foram caracterizados em termos de massa específica real, massa unitária no estado solto e diâmetro máximo característico de acordo com procedimentos estabelecidos nas normas técnicas $[16,17]$. A distribuição de tamanho de partículas dos agregados foi determinada por peneiramento e do resíduo por peneiramento e sedimentação. A morfologia das partículas dos materiais agregados foi observada por microscopia eletrônica de varredura via imagens de elétrons secundários. A análise mineralógica qualitativa da amostra do resíduo de rocha ornamental foi determinada por difração de raios X. A composição química do resíduo em termos de óxidos foi determinada via espectroscopia de raios $\mathrm{X}$ de energia dispersiva (EDX).

$\mathrm{Na}$ atualidade existe a necessidade de se estudar os efeitos relacionados a adição de diferentes minerais com o objetivo de incrementar as propriedades de concretos e argamassas. No presente trabalho é estudada a propriedade da densidade seca aparente máxima. Diversos dos problemas encontrados ao se realizar ensaios são decorrentes da necessidade de estudar simultaneamente o efeito dos fatores (constituintes das misturas) com diferentes níveis. Isto torna os experimentos industriais inviáveis economicamente, visto que os custos e o tempo de execução são elevados [18]. Este trabalho na busca de um modelo mais adequado para as misturas utilizando resíduo de rochas ornamentais, baseouse na metodologia de modelagem numérico experimental rede Simplex (Simplex-Lattice Design) [19-21]. As proporções dos materiais para realização dos ensaios foram calculadas matematicamente, e após o modelo numérico ser estabelecido para a resposta de interesse, seguiu-se à comprovação experimental da eficiência do modelo gerado por meio da Análise de Variância (ANOVA) [22].

No experimento de misturas qualquer variação que ocorra nos componentes, espera-se uma variação proporcional na resposta. Isto é, se as quantidades de todos os componentes da mistura forem triplicadas, a mistura também será triplicada. As proporções dos diversos componentes de uma mistura não são independentes, e obedecem à equação $\mathrm{A}$.

$$
\sum_{i=1}^{\mathrm{q}} \mathrm{X}_{\mathrm{i}}=1,0
$$

na qual q representa o número de componentes da mistura (ou fatores). A representação gráfica desta equação para três componentes $(q=3)$ é apresentada na Fig. 1.

Para a determinação da superfície de resposta da propriedade analisada (densidade seca máxima) foram adotados quatro modelos numéricos, compreendendo a quantidade mínima de pontos para a geração do modelo linear 


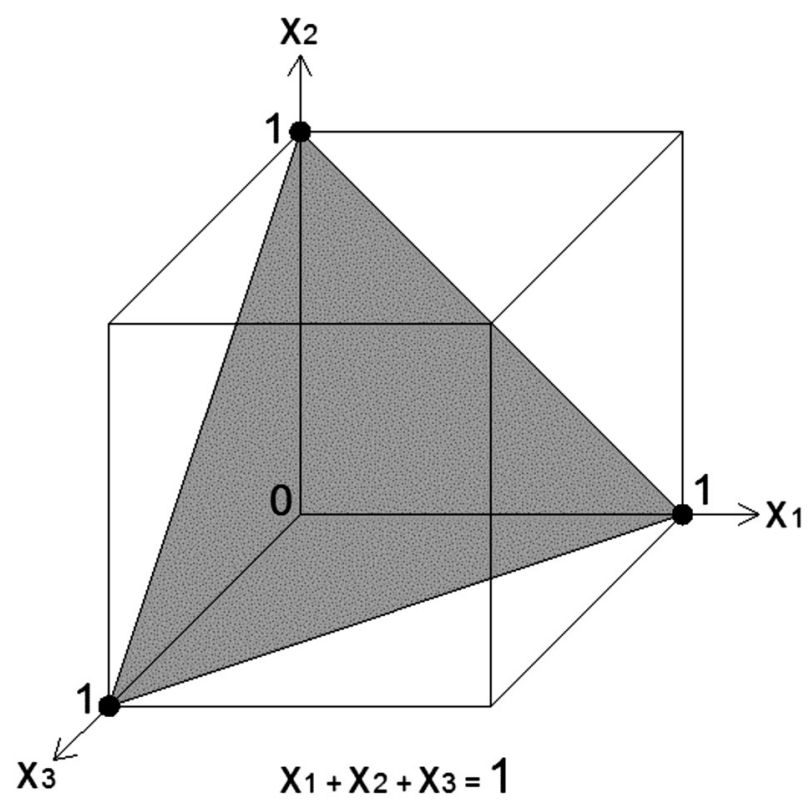

Figura 1: Representação da rede simplex para três componentes (superfície de resposta).

[Figure 1: Representation of the simplex lattice to three components (response surface).]
(3 pontos), modelo quadrático (6 pontos), modelo cúbico simples ( 7 pontos) e modelo cúbico completo (10 pontos). A Fig. 2 ilustra geometricamente os pontos necessários para a obtenção dos modelos matemáticos analisados.

Quando o número de componentes de uma rede é elevado, ou o polinômio tem grau maior que dois, torna-se difícil a representação do sistema de equações. Assim, representa-se o modelo polinomial por uma única equação matricial:

$$
\hat{y}=\mathrm{Xb}
$$

na qual $\hat{y}$ e $b$ são as matrizes contendo respectivamente os valores previstos pelo modelo para $y$ e as estimativas dos parâmetros. $X$ representa a matriz de composição das misturas. Assim sendo, os parâmetros $b$ 's são calculados resolvendo-se apenas uma equação matricial (C), podendo ser facilmente comprovado escrevendo as matrizes por extenso de acordo com:

$$
X^{t} X b=X^{t} y
$$

Isolando-se a matriz $b$ têm-se de forma direta os valores
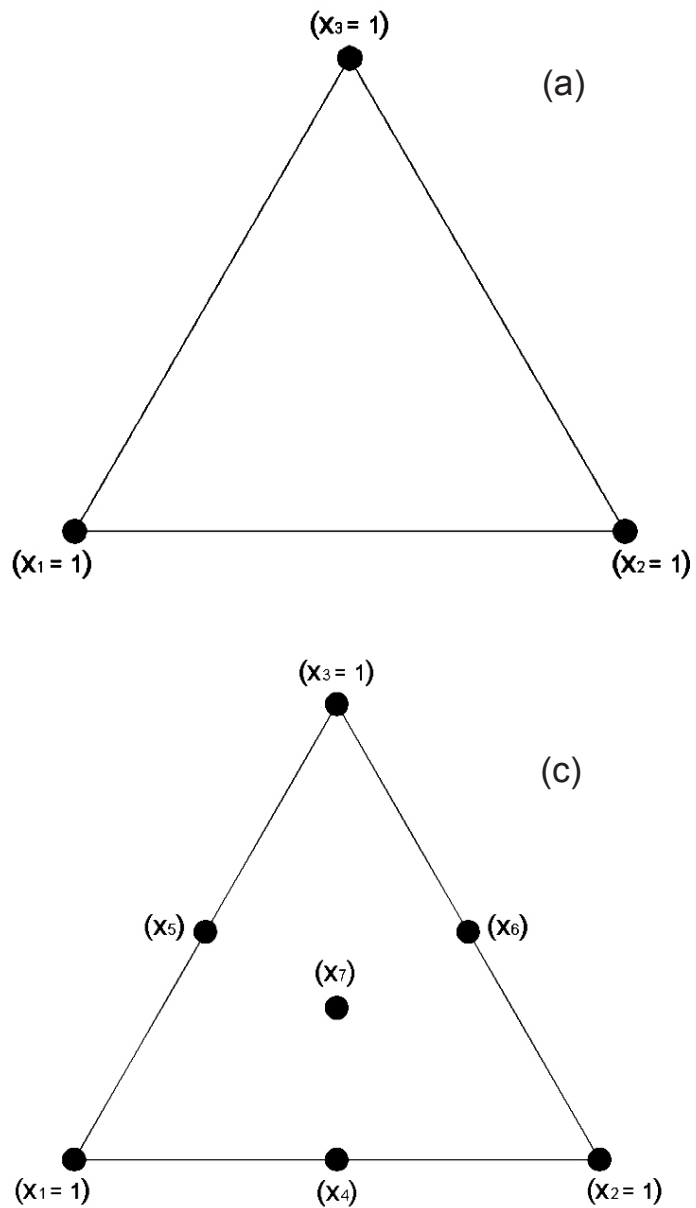
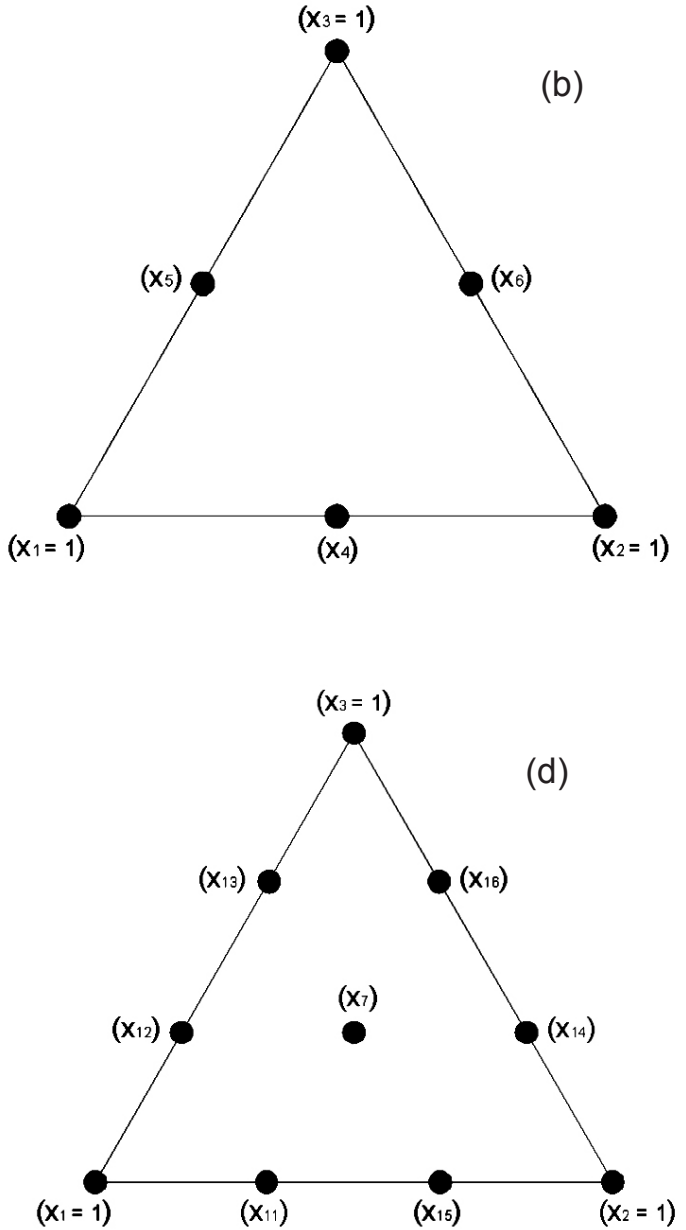

Figura 2: Pontos para obtenção dos modelos testados: a) linear; b) quadrático; c) cúbico simples; e d) cúbico completo. [Figure 2: Points to obtaining of tested models: a) linear; b) quadratic; c) simple cubic; and d) complete cubic.] 
da estimativa dos parâmetros. Se ampliarmos as matrizes $X$ e $y$ adequadamente, tem-se a solução geral para o ajuste de um modelo por mínimos quadrados, não importando quanto sejam as observações ou quantos parâmetros sejam necessários para caracterizar o modelo.

Os corpos-de-prova foram confeccionados com $\mathrm{O}$ molde especificado de acordo com a norma ABNT NBR 12023 [23]. As misturas de brita 0, pó de pedra e resíduo do beneficiamento de rochas ornamentais foram preparadas de acordo com os dezesseis pontos experimentais adotados. Cada mistura foi lançada no molde e realizada vibração em um equipamento Bertel, com vibração de $10 \mathrm{~Hz}$ durante 5 min. Após vibração, foi feito o razamento da mistura com uma régua metálica e pesado para a determinação da densidade seca aparente.

\section{RESULTADOS E DISCUSSÃO}

Os resultados da composição química do resíduo de rocha ornamental encontram-se na Tabela I. Os constituintes determinantes são sílica $\left(\mathrm{SiO}_{2}\right)$, alumina $\left(\mathrm{Al}_{2} \mathrm{O}_{3}\right)$ e óxido de potássio $\left(\mathrm{K}_{2} \mathrm{O}\right)$, nessa ordem. $\mathrm{O}$ alto teor de sílica indica que o resíduo é oriundo de rochas silicatas ácidas, provavelmente granitos e gnaisses. Além disso, o baixo teor de óxido de ferro $\left(\mathrm{Fe}_{2} \mathrm{O}_{3}\right)$ e óxido de cálcio $(\mathrm{CaO})$ no resíduo estudado estão fundamentalmente relacionados ao processo de polimento das rochas ornamentais em que são utilizados abrasivos. Análise mineralógica qualitativa por difração de raios $\mathrm{X}$ indicou as presenças das seguintes fases cristalinas: sílica, feldspato potássico, mineral micáceo, hematita e calcita, o qual corrobora os dados de composição química.

Nas Figs. 3a-d são apresentados os aspectos morfológicos das partículas do resíduo de rocha ornamental utilizado neste trabalho. Na Fig. 3a são observados detalhes dos cristais de feldspato com seu típico hábito prismático. Na Fig. 3b observa-se um cristal de mica com seu típico hábito planar.

Tabela I - Composição química do resíduo de rocha ornamental (\%).

[Table I - Chemical composition of the ornamental rock waste (\%).]

\begin{tabular}{lc}
\hline $\mathrm{SiO}_{2}$ & 67,638 \\
$\mathrm{Al}_{2} \mathrm{O}_{3}$ & 16,416 \\
$\mathrm{Fe}_{2} \mathrm{O}_{3}$ & 2,994 \\
$\mathrm{~K}_{2} \mathrm{O}$ & 7,552 \\
$\mathrm{CaO}$ & 3,337 \\
$\mathrm{SO}_{3}$ & 1,425 \\
$\mathrm{TiO}_{2}$ & 0,424 \\
$\mathrm{Sc}_{2} \mathrm{O}_{3}$ & 0,098 \\
$\mathrm{MnO}$ & 0,050 \\
$\mathrm{Rb}_{2} \mathrm{O}$ & 0,020 \\
$\mathrm{ZnO}$ & 0,017 \\
$\mathrm{ZrO}$ & 0,017 \\
$\mathrm{SrO}$ & 0,011 \\
\hline
\end{tabular}
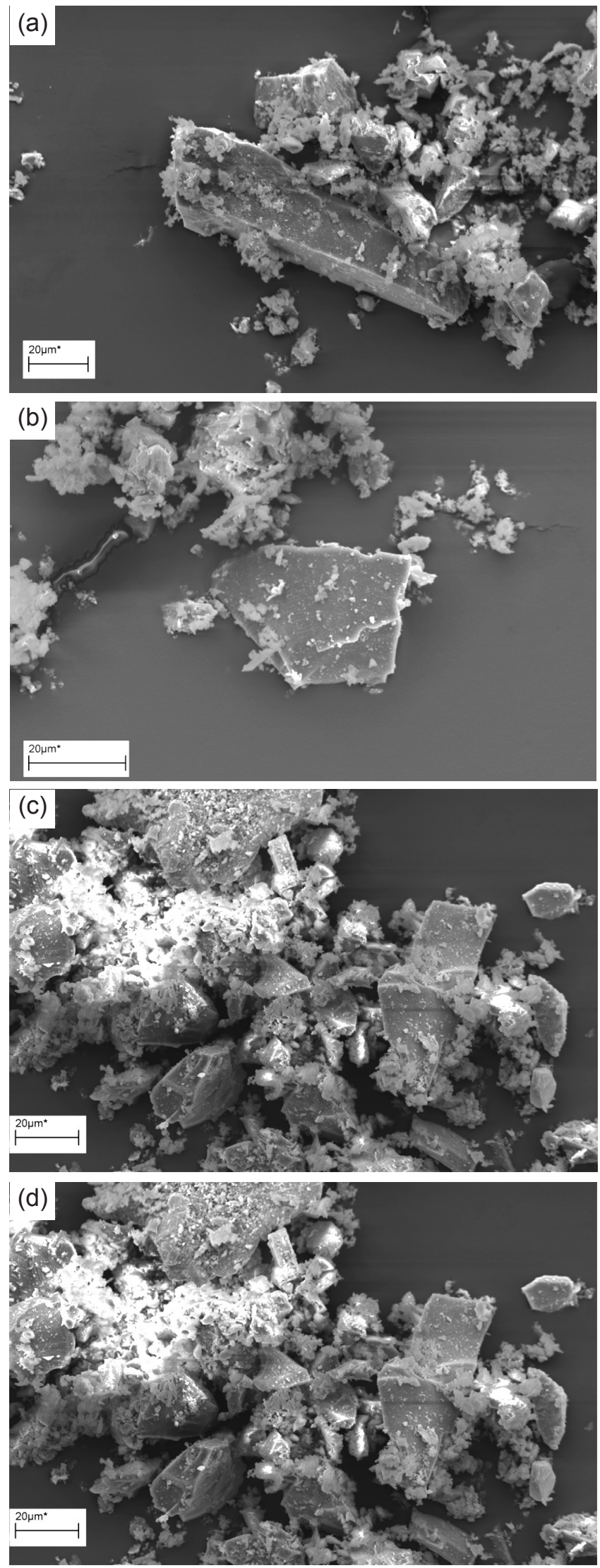

Figura 3: Aspectos morfológicos das partículas do resíduo de rocha ornamental.

[Figure 3: Morphological aspects of the particles of the ornamental rock waste.] 
Na Fig. 3c são observados detalhes do cristal de quartzo com a presença de fraturas conchoidais. Na Fig. 3d têmse aglomerados de minerais com as presenças de quartzo, feldspato e mica.

Na Fig. 4 são apresentadas as curvas granulométricas dos agregados utilizados no planejamento experimental. Em termos de tamanho de partículas, os materiais agregados são bastante diferentes. Verifica-se que o resíduo de rocha ornamental apresentou granulometria mais fina, o qual pode ser usado como um material filler $(\sim 95 \%<75 \mu \mathrm{m})$. Este resultado é importante devido às partículas mais finas do resíduo, em princípio, poderem preencher os vazios entre as partículas maiores. Isto pode contribuir para otimizar o empacotamento das partículas e, portanto, maximizar a densidade seca aparente de materiais agregados industriais na produção de concretos convencionais.

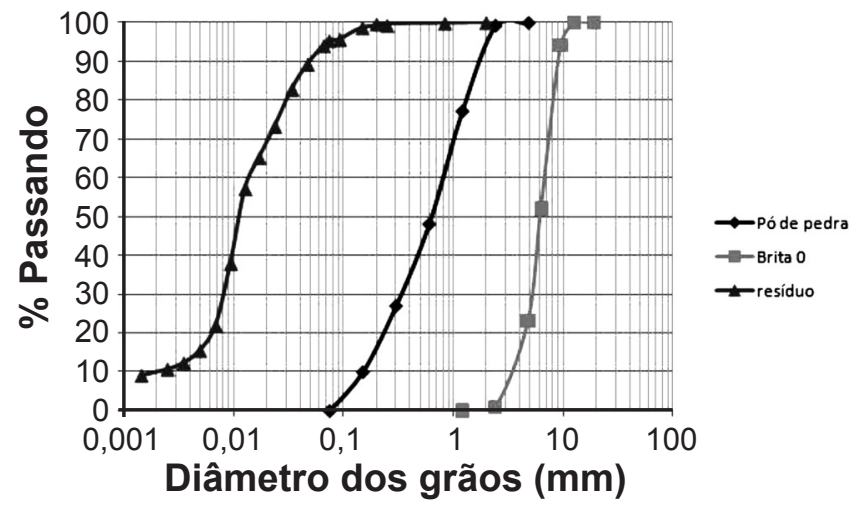

Figura 4: Curvas de distribuição de partículas dos materiais usados no planejamento experimental.

[Figure 4: Particle size distribution curves of the materials used in the experimental design.]

A Tabela II apresenta as características físicas dos materiais utilizados na composição das misturas. Os materiais apresentam valores de massa específica real relativamente próximos na faixa entre $2,65-2,77 \mathrm{~g} / \mathrm{cm}^{3}$. Pode-se observar que em termos de massa unitária no estado solto, o resíduo de rocha ornamental apresenta o menor empacotamento das partículas. Isto se deve fundamentalmente a sua granulometria mais fina, como

Tabela II - Caracterização física dos materiais agregados utilizados.

[Table II - Physical characterization of the agregate materials used.]

\begin{tabular}{lccc}
\hline Propriedade & Brita 0 & $\begin{array}{l}\text { Pó de } \\
\text { pedra }\end{array}$ & Resíduo \\
\hline $\begin{array}{l}\text { Massa específica } \\
\text { real, g/cm }\end{array}$ & 2,77 & 2,72 & 2,65 \\
$\begin{array}{l}\text { Massa específica no } \\
\text { estado solto, g/cm }\end{array}$ & 1,44 & 1,48 & 0,98 \\
$\begin{array}{l}\text { Diâmetro máximo } \\
\text { característico, mm }\end{array}$ & 9,50 & 2,40 & 0,075 \\
\hline
\end{tabular}

observado na Fig. 4. Em termos de diâmetro máximo característico os materiais são bem diferentes, sendo que a brita 0 é classificada como agregado graúdo e o pó de pedra como agregado miúdo. Já o resíduo de rocha ornamental utilizado pode ser classificado como um material filler.

\section{Planejamento de experimentos em rede simplex}

Na Fig. 5 estão representados os pontos experimentais utilizados na composição das misturas e determinação da superfície de resposta. Verifica-se que são 16 pontos experimentais usados no planejamento experimental para determinação da densidade seca aparente máxima das misturas. A expansão das matrizes representadas na equação (B) foi feita para obtenção das equações dos modelos com seus respectivos parâmetros $b$ calculados. $\mathrm{Na}$ Tabela III são apresentadas as nomenclaturas de respostas usadas para misturas dos três componentes (brita 0 , pó de pedra e resíduo de rocha ornamental).

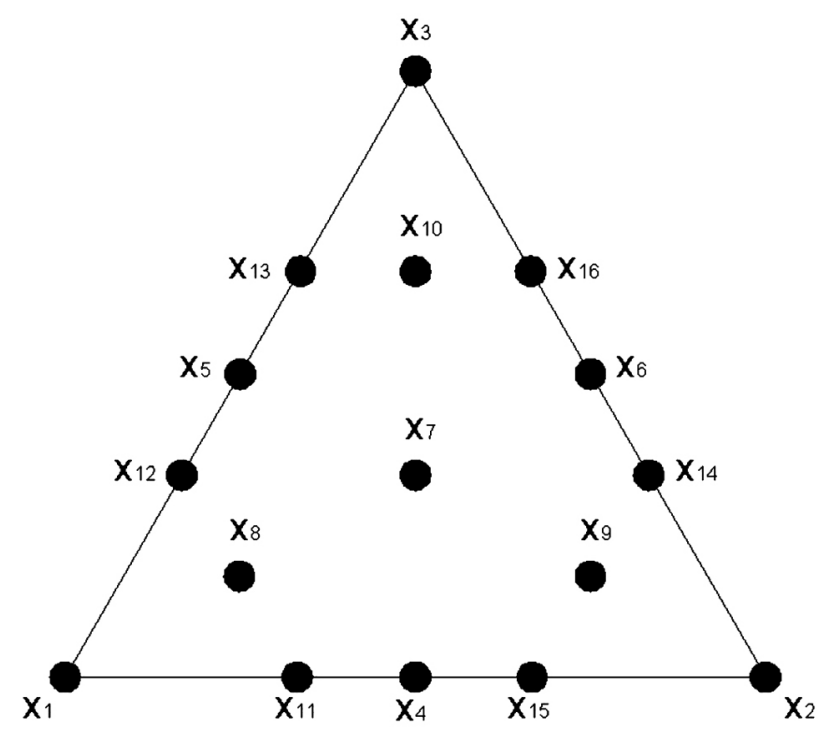

Figura 5: Pontos experimentais para determinação de densidade seca aparente.

[Figure 5: Experimental points for determination of the apparent dry density.]

A partir da matriz de composição das misturas (Tabela III) foram gerados por meio de planejamento experimental os seguintes modelos matemáticos: modelo linear, modelo quadrático, modelo cúbico simples e modelo cúbico completo. Na Tabela IV são apresentados os resultados da análise de variância (ANOVA) para os modelos testados. Verifica-se que o modelo cúbico completo apresentou o maior coeficiente de determinação $\left(\mathrm{R}^{2}\right)$, que foi da ordem de $96,66 \%$. Quanto mais próximo da unidade estiver o valor de $\left(\mathrm{R}^{2}\right)$, melhor será o ajuste do modelo aos dados observados. Isto significa que esse modelo apresenta maior homogeneidade e maior confiabilidade. De forma que o modelo cúbico completo será usado daqui por diante para determinação da superfície de resposta para a densidade 
Tabela III - Matriz de composição das misturas dos pontos experimentais.

[Table III - Matrix composition of mixtures of experimental points.]

\begin{tabular}{|c|c|c|c|c|}
\hline \multirow{2}{*}{$\begin{array}{c}\text { Pontos } \\
\text { experimentais }\end{array}$} & \multicolumn{3}{|c|}{$\begin{array}{l}\text { Matriz de composição das } \\
\text { misturas - X }\end{array}$} & \multirow{2}{*}{$\begin{array}{l}\text { Respostas } \\
\quad(\bar{y})- \\
\text { Matriz das } \\
\text { respostas - y }\end{array}$} \\
\hline & Brita 0 & $\begin{array}{l}\text { Pó de } \\
\text { pedra }\end{array}$ & Residuo & \\
\hline $\mathrm{X}_{1}$ & 1 & 0 & 0 & $\overline{y_{1}}$ \\
\hline$x_{2}$ & 0 & 1 & 0 & $\mathrm{y}_{2}$ \\
\hline$x_{3}$ & 0 & 0 & 1 & $y_{3}$ \\
\hline $\mathrm{x}_{4}$ & $1 / 2$ & $1 / 2$ & 0 & $\mathrm{y}_{4}$ \\
\hline $\mathrm{x}_{5}$ & $1 / 2$ & 0 & $1 / 2$ & $\mathrm{y}_{5}$ \\
\hline$x_{6}$ & 0 & $1 / 2$ & $1 / 2$ & $\mathrm{y}_{6}$ \\
\hline$x_{7}$ & $1 / 3$ & $1 / 3$ & $1 / 3$ & $\mathrm{y}_{7}$ \\
\hline $\mathrm{x}_{8}$ & $2 / 3$ & $1 / 6$ & $1 / 6$ & $\mathrm{y}_{8}$ \\
\hline$x_{9}$ & $1 / 6$ & $2 / 3$ & $1 / 6$ & $\mathrm{y}_{9}$ \\
\hline $\mathrm{x}_{10}$ & $1 / 6$ & $1 / 6$ & $2 / 3$ & $\mathrm{y}_{10}$ \\
\hline$x_{11}$ & $2 / 3$ & $1 / 3$ & 0 & $\mathrm{y}_{11}$ \\
\hline $\mathrm{x}_{12}^{11}$ & $2 / 3$ & 0 & $1 / 3$ & $\mathrm{y}_{12}$ \\
\hline $\mathrm{x}_{13}$ & $1 / 3$ & $2 / 3$ & 0 & $\mathrm{y}_{13}$ \\
\hline $\mathrm{x}_{14}$ & 0 & $2 / 3$ & $1 / 3$ & $\mathrm{y}_{14}$ \\
\hline $\mathrm{X}_{15}$ & $1 / 3$ & 0 & $2 / 3$ & $\mathrm{y}_{15}$ \\
\hline $\mathrm{x}_{16}$ & 0 & $1 / 3$ & $2 / 3$ & $\mathrm{y}_{16}$ \\
\hline
\end{tabular}

Tabela IV - ANOVA para os modelos testados.

[Table IV - Tested models ANOVA.]

\begin{tabular}{ccccc}
\hline & & \multicolumn{3}{c}{ Modelos Testados } \\
\cline { 3 - 5 } Parâmetros & Linear & Quadrático & $\begin{array}{c}\text { Cúbico } \\
\text { Simples }\end{array}$ & $\begin{array}{c}\text { Cúbico } \\
\text { Completo }\end{array}$ \\
\hline $\mathrm{SQ}_{\mathrm{R}}$ & 0,636 & 1,437 & 1,471 & 1,620 \\
$\mathrm{G} . \mathrm{L}$ & 2 & 5 & 6 & 8 \\
$\mathrm{SQ}$ & 1,676 & 1,676 & 1,676 & 1,676 \\
$\mathrm{G} . \mathrm{L}$ & 47 & 47 & 47 & 47 \\
$\mathrm{R}^{2}(\%)$ & 37,95 & 85,74 & 87,77 & 96,66 \\
\hline
\end{tabular}

$S Q_{R}=$ soma quadrática de regressão; $S Q_{T}=$ soma quadrática total;

$G . L=$ graus de liberdade e $R^{2}=\%$ de variação explicável .

seca aparente das misturas.

Na Fig. 6 é apresentado o diagrama de Pareto para o modelo cúbico completo. Este diagrama mostra de forma eficiente e rápida os efeitos que são estatisticamente importantes. Neste caso os efeitos cujos retângulos estiverem à direita da linha divisória $(\mathrm{p}=0,5)$ devem ser considerados no modelo matemático. Portanto, a equação para o modelo cúbico completo é dada por:

$\hat{y}=1,58 x_{1}^{\prime}+1,66 x_{2}^{\prime}+1,28 x^{\prime}{ }_{3}+0,92 x_{1}^{\prime} x_{2}^{\prime}+$

$1,41 x_{1}{ }_{1} x_{3}+1,00 x^{\prime}{ }_{2} x^{\prime}{ }_{3}+2,76 x^{\prime}{ }_{1} x_{2}{ }_{2} x_{3}+$

$1,20 x_{1}^{\prime} x_{3}^{\prime}{ }_{3}\left(x_{1}^{\prime}{ }_{1}-x_{3}^{\prime}\right)++1,30 x_{2}^{\prime} x^{\prime}{ }_{3}\left(x_{2}^{\prime}{ }_{2}-x_{3}^{\prime}\right)$

A Tabela $\mathrm{V}$ mostra o comparativo entre os valores obtidos nos experimentos $(\overline{\mathrm{y}})$ e os valores estimados pelo modelo cúbico completo $(\hat{y})$, calculados pela equação $\mathrm{D}$.

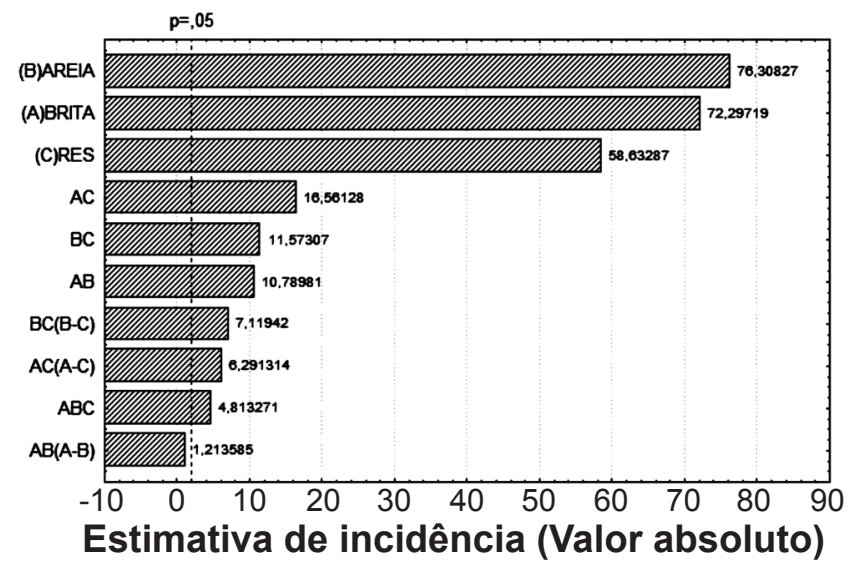

Figura 6: Diagrama de Pareto para o modelo cúbico completo. [Figure 6: Pareto diagram for the complete cubic model.]

Tabela V - Comparação entre os valores observados e previstos pelo modelo cúbico completo.

[Table V-Comparison between the observed and predicted values by the complete cubic model.]

\begin{tabular}{|c|c|c|c|c|}
\hline$X_{1}$ & $X_{2}$ & $X_{3}$ & $\begin{array}{c}\bar{y} \\
\text { (observado) }\end{array}$ & $\begin{array}{c}\hat{y} \\
\text { cúbico } \\
\text { completo }\end{array}$ \\
\hline 1 & 0 & 0 & 1,58 & 1,58 \\
\hline 0 & 1 & 0 & 1,66 & 1,66 \\
\hline 0 & 0 & 1 & 1,27 & 1,28 \\
\hline $1 / 2$ & $1 / 2$ & 0 & 1,85 & 1,85 \\
\hline $1 / 2$ & 0 & $1 / 2$ & 1,77 & 1,78 \\
\hline 0 & $1 / 2$ & $1 / 2$ & 1,72 & 1,72 \\
\hline $1 / 3$ & $1 / 3$ & $1 / 3$ & 1,94 & 1,98 \\
\hline $2 / 3$ & $1 / 6$ & $1 / 6$ & 2,03 & 1,95 \\
\hline $1 / 6$ & $2 / 3$ & $1 / 6$ & 1,99 & 1,96 \\
\hline $1 / 6$ & $1 / 6$ & $2 / 3$ & 1,58 & 1,60 \\
\hline $2 / 3$ & $1 / 3$ & 0 & 1,81 & 1,81 \\
\hline $2 / 3$ & 0 & $1 / 3$ & 1,85 & 1,88 \\
\hline $1 / 3$ & $2 / 3$ & 0 & 1,83 & 1,84 \\
\hline 0 & $2 / 3$ & $1 / 3$ & 1,84 & 1,85 \\
\hline $1 / 3$ & 0 & $2 / 3$ & 1,64 & 1,60 \\
\hline 0 & $1 / 3$ & $2 / 3$ & 1,55 & 1,53 \\
\hline \multicolumn{3}{|c|}{$\begin{array}{l}\text { Soma dos resíduo } \\
\text { deixado pelo modelo } \\
\text { (valor que não } \\
\text { entra no cálculo do } \\
\text { modelo) }\end{array}$} & $\sum \hat{\mathrm{e}}$ & 0,30 \\
\hline \multicolumn{3}{|c|}{$\begin{array}{l}\text { Média dos resíduos } \\
\text { experimentais }\end{array}$} & $\bar{e}$ & 0,02 \\
\hline
\end{tabular}

Nas Figs. 7a-b é apresentado o comportamento estatístico para a densidade seca aparente. Na Fig. 7a tem-se a relação entre os valores previstos versus valores observados. Verifica-se uma relação praticamente linear, o qual caracteriza um ajuste satisfatório. Na Fig. $7 \mathrm{~b}$ tem-se 

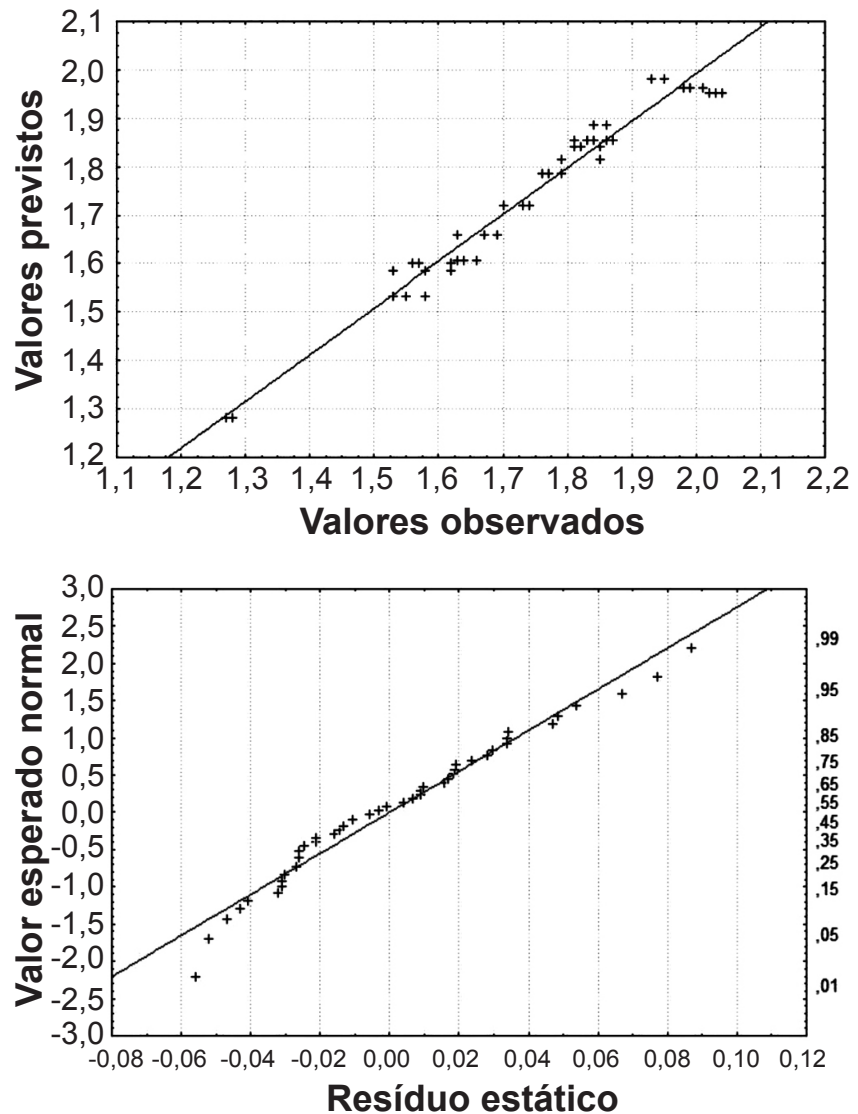

Figura 7: Comportamento estatístico da densidade seca aparente: a) valores previstos versus valores observados; e b) curva da probabilidade normal.

[Figure 7: Statistical behavior of the apparent dry density: a) prescribed values versus observed values; and b) Curve of normal probability.]

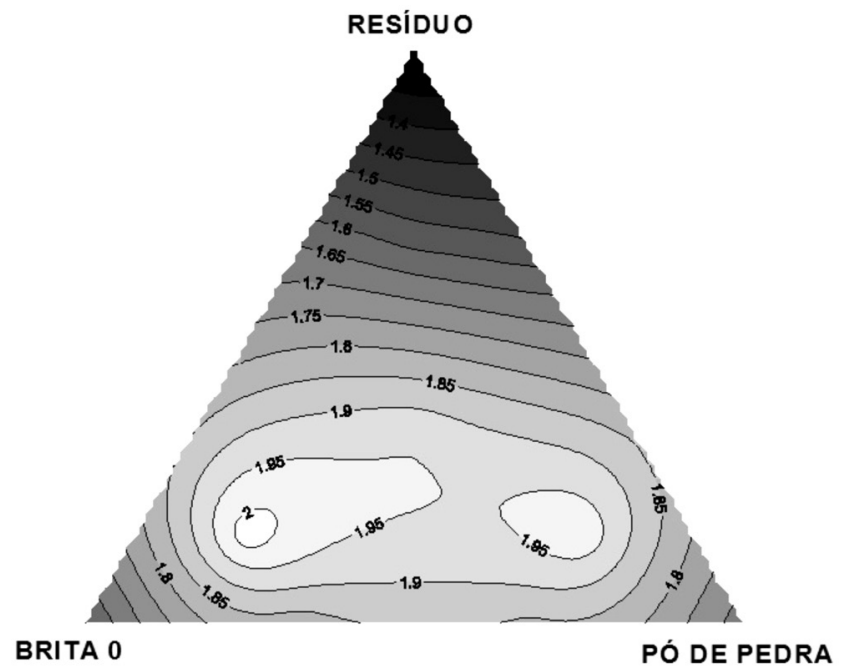

Figura 8: Superfície de resposta obtida pelo modelo cúbico completo para a densidade seca aparente.

[Figure 8: Response surface obtained by the complete cubic model for apparent dry density.]

o comportamento dos resíduos estatísticos para o modelo adotado. Observa-se que a curva dos resíduos no gráfico de probabilidade normal se aproxima de uma linha reta, o que indica que a suposição de normalidade para o modelo está satisfeita.

A Fig. 8 apresenta a superfície de resposta obtida por meio do modelo cúbico completo, tanto para o teste $\mathrm{F}$ quanto para a média dos resíduos experimentais, para os dados medidos para a propriedade analisada (densidade seca aparente). Nesta figura os componentes brita 0 , pó de pedra e resíduo de rocha ornamental representados nos vértices do triângulo de superfície de resposta correspondem às proporções máximas de $100 \%$ respectivamente em massa de cada elemento da mistura. Considera-se que se a razão entre as médias quadráticas (regressão $\left(\mathrm{MQ}_{\mathrm{R}}=0,2024\right)$ e resíduos $\left.\left(\mathrm{MQ}_{\mathrm{r}}=0,0015\right)\right)$ for maior que o valor do teste $\mathrm{F}$ tabelado, então existe relação linear entre as variáveis y e x. Para que uma regressão não seja apenas estatisticamente significativa, mas também útil para preditivos, o valor da relação entre as médias quadráticas (regressão e resíduos) deve ser comparado com no mínimo quatro a cinco vezes o valor do teste $\mathrm{F}$ tabelado $\left(\mathrm{F}_{8,49}=2,18\right)[23]$, como mostrado na expressão E.

$\frac{\mathrm{MQ}_{\mathrm{R}}}{\mathrm{MQ}_{\mathrm{r}}}>5 \mathrm{~F}_{\mathrm{tab}} \rightarrow \frac{0,2024}{0,0015}=134,93>5 \times(2,18)=10,90$

Os resultados gerados pela superfície de resposta indicam que a região de maior compacidade para as misturas dos materiais filler, corresponde ao valor de densidade seca aparente máxima de $2,0 \mathrm{~g} / \mathrm{cm}^{3}$. Para esta região, a composição ternária com maior porcentagem de resíduo na mistura corresponde a: $63 \%$ de brita $0+17 \%$ de pó de rocha $+20 \%$ de resíduo de rocha ornamental.

\section{CONCLUSÕES}

A utilização do planejamento experimental em rede simplex mostrou-se uma ferramenta viável para avaliar a densidade seca aparente máxima de traços com agregados industrializados contendo resíduo de rocha ornamental como um material filler. Os resultados mostraram que o modelo cúbico completo é o que melhor se ajusta aos pontos experimentais para determinação da superfície de resposta. De acordo com a superfície de resposta gerada a região de maior compacidade corresponde aquela com densidade seca aparente de $2,0 \mathrm{~g} / \mathrm{cm}^{3}$. Esta região corresponde ao traço 63\% de brita $0: 17 \%$ de pó de pedra: $20 \%$ de resíduo de rocha ornamental. Portanto, o resíduo do beneficiamento de rochas ornamentais pode ser utilizado como um material filler em substituição parcial a agregados industrializados para a construção civil. Esta é uma alternativa de reciclagem para os resíduos gerados no setor de rochas ornamentais viável, atual e de elevada importância econômica e ambiental.

\section{AGRADECIMENTOS}

Ao CNPq e à FAPERJ pelo apoio financeiro, à empresa Decolores Mármores e Granitos pelo fornecimento do resíduo de rocha ornamental, e à empresa Pedreira Itereré Ind. Com. 
pelo fornecimento da brita 0 e pó de pedra.

\section{REFERÊNCIAS}

[1] ASM, ASM Handbook - Metallurgy Powder, $9^{\text {th }}$ ed., v. 7, ASM International, EUA (1984).

[2] ASM, Eng. Mater. Handbook - Ceramics and Glass, v. 4, ASM International, EUA (1984).

[3] I. R. Oliveira,A.R. Studart, R. G. Pileggi, V.C. Pandolfelli, Dispersão e empacotamento de partículas, $2^{\mathrm{a}}$ ed., Fazendo Arte Editorial, S. Paulo, SP (2000) 224 p.

[4] P. K. Metha, P. J. M. Monteiro, Concreto: estrutura, propriedades e materiais, PINI, S. Paulo, SP (1994) 573p.

[5] A. M. Neville, Propriedades do Concreto, Trad.: S. E. Giammusso, $2^{\mathrm{a}}$ ed., PINI, S. Paulo, SP (1997) 828p.

[6] Associação Brasileira da Indústria de Rochas Ornamentais, Situação atual e perspectivas brasileiras no setor de rochas ornamentais, Informe 002/2008, S. Paulo, SP (2008).

[7] R. R. Menezes, H. S. Ferreira, G. A. Neves, H. C. Ferreira, Cerâmica 48, 306 (2002) 92-101.

[8] P. Torres, H. R. Fernandes, S. Agathopoulos, D. U. Tulyaganov, J. H. F. Ferreira, J. Eur. Ceram. Soc. 24 (2004) 3177-3185.

[9] M. H. B. O. Frasca, Rocha como material de construção, in Materiais de construção civil e princípios de ciência dos materiais, v.1, IBRACON, S. Paulo, SP (2007) 437-480.

[10] J. P. V. T. Manhães, J. N. F. Holanda, Química Nova 31, 6 (2008) 1301-1304.

[11] J. M. S. Moreira, J. P. V. T. Manhães, J. N. F. Holanda, Cerâmica 51, 319 (2005) 180-186.

[12] A. M. Segadães, M. A. Carvalho, W. Acchar, Appl. Clay
Sci. 30, 1 (2005) 42-52.

[13] F. Saboya Jr., G. C. Xavier, J. Alexandre, Const. Build. Mater. 21, (2007) 1950-1960.

[14] A. Z. Destefani, Diss. Mestrado, UENF-PPGEC, Campos dos Goytacazes, RJ (2009).

[15] ABNT, Associação Brasileira de Normas Técnicas, NBR 10007: Amostragem de resíduos sólidos, Rio de Janeiro, RJ (2004).

[16] ABNT, Associação Brasileira de Normas Técnicas, NBR 7211: Agregados para concreto - especificação, Rio de Janeiro, RJ (1983).

[17] ABNT, Associação Brasileira de Normas Técnicas, NBR 7251: Agregados em estado solto - determinação da massa unitária: método de ensaio, Rio de Janeiro, RJ (1982).

[18] E. V.C. Galdamez,L.C. R. Carpinetti, Gestão e Produção 11, 1 (2004) 121-134.

[19] J. A. Cornell, Experiments with mixtures: designs, models, and the analysis of mixture data, $2^{\text {nd }}$ ed., John Wiley \& Sons (1990).

[20] B. Barros Neto, I. S. Spacino, R. E. Bruns, Como fazer experimentos: pesquisa e desenvolvimento na indústria, $2^{\mathrm{a}}$ ed., Editora da Unicamp, Campinas, SP (2003).

[21] A. V. Bahiense, R. T. Manhães, J. Alexandre, G. C. Xavier, S. N. Monteiro, C. M. F. Viera, Cerâmica 54, 332 (2008) 395-403.

[22] V. Calado, D. Montgomery, Planejamento de experimentos usando o statistica, E-papers Serviços Editoriais, Rio de Janeiro, RJ (2003) 260p.

[23] ABNT, Associação Brasileira de Normas Técnicas, NBR 12023: Solo-cimento - ensaio de compactação, Rio de Janeiro, RJ (1992).

(Rec.03/09/2010, Rev.26/12/2010, Ac. 14/02/2011) 